\title{
Ingresos económicos de mujeres frente a hombres por actividad económica en México
}

\section{Economic income of women compared to men by economic activity in Mexico}

\author{
Miriam Edith Pérez-Romero 1, 2 (1) 0000-0003-1450-9644 \\ Martha Beatriz Flores-Romero ${ }^{1}$ (D) 0000-0003-4567-5485 \\ Donají Jiménez-Islas ${ }^{2}$ (D) 0000-0001-8227-2525 \\ ${ }^{1}$ Universidad Michoacana de San Nicolás de Hidalgo ${ }^{\natural}$ miromero@umich.mx \\ ${ }^{2}$ Instituto Tecnológico Superior de Huichapan \\ (C) Universidad De La Salle Bajío (México)
}

Palabras clave: actividades económicas; SCIAN; ingreso; discriminación salarial; brecha salarial; descomposición Oaxaca-Blinder, Mincer; economía; género; desigualdad; desigualdad económica; desigualdad laboral; igualdad de género; México

Keywords: economic activities; SCIAN; income; wage discrimination; wage gap; decomposition OaxacaBlinder, Mincer; economy; gender; inequality; economic inequality; labor inequality; gender equality; Mexico

Recepción: 17 - 12 - 2020 / Aceptación: 28 - 05 - 2021

\section{Resumen}

Introducción: la desigualdad laboral que viven hombres y mujeres se hace evidente en los ingresos. El objetivo del presente trabajo fue analizar los ingresos económicos de mujeres y hombres en las actividades económicas en México durante los últimos 15 años, a partir de la descomposición Oaxaca-Blinder, con el propósito de detectar las brechas salariales por género.

Método: para llevar a cabo el análisis, se consideraron los 20 sectores de actividad contemplados en el Sistema de Clasificación Industrial de América del Norte. Se extrajo información de la Encuesta Nacional de Ocupación y Empleo acerca del sexo, los ingresos por hora, los años de escolaridad y la edad de los individuos. Se aplicó la ecuación básica de descomposición OaxacaBlinder posterior a la realización de análisis econométricos mediante regresiones lineales múltiples, a través de la ecuación de salarios de Mincer.

Resultados: los resultados del análisis presentan una brecha salarial en las actividades económicas de, por un lado, a) construcción, y b) transporte, comercio y almacenamiento, favorable a las mujeres en cada uno de los años del análisis. Por otro lado, en las actividades c) corporativos, y d) otros servicios, excepto actividades gubernamentales, se aprecia una brecha salarial favorable a los hombres en todos los años del estudio. 
Discusión o conclusión: los ingresos económicos han permitido mostrar la diferencia salarial entre hombres y mujeres en las distintas actividades económicas de México, favorable en algunas actividades a las mujeres, aunque en la mayoría es favorable a los hombres.

\begin{abstract}
Introduction: the labor inequality experienced by women and men is evident in income. The objective of this work was to analyze the economic income of women and men in economic activities in Mexico, during the last 15 years, based on the Oaxaca-Blinder decomposition, in order to detect wage gaps by gender.

Method: to carry out the analysis, the 20 activity sectors contemplated in the North American Industrial Classification System were considered. Information was extracted from the National Survey of Occupation and Employment about the sex, hourly income, years of schooling and age of the individuals. The basic decomposition equation Oaxaca-Blinder was applied after conducting econometric analysis through multiple linear regressions through Mincer's wage equation.

Results: the results of the analysis show a wage gap in the economic activities of, on the one hand a) construction, and b) transportation, commerce, and storage, favorable to women in each of the years of the analysis. On the other hand, in the activities of c) corporate, and d) other services, except activities government, it is appreciated a wage gap favorable to men in all years of the study. Discussion or Conclusion: the economic income has allowed showing the salary difference between men and women in the different economic activities of Mexico, favorable in some activities to women although in the majority it is favorable to men.
\end{abstract}

\title{
Introducción
}

La ppromoción de la igualdad plena de género y la eliminación de la discriminación, así como la integración y participación femenina en el desarrollo, se establecieron en la primera Conferencia Mundial sobre la Mujer celebrada en 1975 por la Organización de las Naciones Unidas (ONU), (Saavedra y Camarena, 2020). En esa misma década, se dio la incorporación masiva de mujeres al mercado laboral, lo que representó a nivel global un cambio económico y social, visibilizando la fuerza productiva femenina a través del trabajo extradoméstico (Zabludovsky, 2007; Pedrero, 2004).

No 27, Vol. 13 (3), 2021. ISSN $2007-0705$, pp.: 1 - 19

- 2 - 
Actualmente, las mujeres abarcan el $40 \%$ de la mano de obra a nivel mundial (De la Torre et al., 2020); específicamente en América Latina se observa un crecimiento sostenido de la participación laboral femenina, a un ritmo superior al de los hombres (Abramo, 2004).

Hablar de la mujer en México es referirse a más de la mitad de la población total: de acuerdo con el Censo de Población y Vivienda 2020 realizado por el Instituto Nacional de Estadística y Geografía (INEGI), hay 95.2 hombres por cada 100 mujeres. Ahora bien, si esto se puntualiza sobre el contexto laboral, existen 40506343 hombres y 43157097 mujeres en edad de trabajar; en este caso la relación hombres - mujeres es de 93.9 - 100.

En la última década, las mujeres han mostrado una creciente participación en la vida social, económica, política y cultural de México (Moctezuma et al., 2014). Sin embargo, las disparidades de género siguen siendo considerables, ya que perduran barreras invisibles en cuanto a los salarios y el acceso a puestos directivos. Por otro lado, las mujeres están representadas principalmente en sectores económicos de baja productividad o de economía informal (Saavedra y Camarena, 2020), por lo que, ante esas barreras y escenarios, la condición de ser mujer es un elemento de desventaja (Rodríguez-Pérez et al., 2017).

Las situaciones y fenómenos que llevan a las mujeres a incorporarse al mercado laboral persisten; uno de esos fenómenos es la continuidad y agudización de los problemas económicos del país (Sosa y Román, 2015). En una comparación entre los ingreso per cápita, la inflación y el PIB de los años 2010 y 2011 se identificó que no son proporcionales al crecimiento de la población, lo que se traduce en baja producción de bienes y servicios, menor ahorro e inversión, y bajo ingreso per cápita que, a su vez, genera un bajo nivel de vida, así como una balanza comercial desfavorable (Cardona et al., 2018).

La discriminación de género se refiere a la disparidad de condiciones experimentadas entre hombres y mujeres por el sólo hecho de pertenecer a uno u otro sexo, fenómeno que se observa en diversos ámbitos de la vida social (Bucio, 2014). En el ámbito económico, una forma de medir la desigualdad de género es a través de la brecha salarial o discriminación salarial (Bakas et al., 2018). La discriminación salarial acontece cuando un individuo con similares características a otro, y que solamente difiere en sexo, recibe menor salario por razones inexplicables distintas al desempeño de su trabajo (Cadena, 2020).

Diversos estudios han abordado la brecha de género o la desigualdad laboral. Entre ellos, el de Flores y Salas (2015), quienes relacionaron la brecha de género con la calidad del empleo en 
México: la variable más significativa es la educación superior, ya que a partir de los estudios profesionales se comienzan a invertir las brechas de género, situación más evidente a nivel posgrado, donde las mujeres obtienen mejores condiciones laborales que los hombres. Como segundo aspecto, los adultos jóvenes hombres cuentan con una mayor estabilidad laboral y más prestaciones, pero las mujeres cuentan con una mayor relación salario-tiempo de dedicación.

Martínez y Acevedo (2004), reportaron que el 85 \% de la discriminación es resultado del efecto de la estructura salarial vigente en el mercado de trabajo, el $15 \%$ restante se explica por una mayor productividad marginal de la mujer en comparación con hombres, lo cual se refleja en el salario que recibe.

Camarena et al. (2015) abordaron aspectos de legislación, desigualdad de sexo en la enseñanza, participación de la mujer en el mercado laboral, participación de la mujer en la vida política y el género en el emprendimiento. Sus hallazgos fueron que la pobreza afecta con mayor intensidad a las mujeres por la falta de autonomía económica, marcada brecha en el ámbito económico y políticos; además, las mujeres emprenden ante la imposibilidad de ascender en su carrera laboral en las organizaciones.

Rodríguez-Pérez et al. (2017), describen la brecha salarial por género, en el sector público es favorable para las mujeres y en el sector privado las desfavorece, particularmente en el sector informal.

Ante los fenómenos sociales como el tema de género, la economía mexicana no está exenta a la trasformación de su modelo de desarrollo, está pasando de ser una economía cerrada a un modelo con fuerte orientación hacia el exterior y donde las fuerzas del mercado tienen una mayor presencia, por tanto se requiere determinar el impacto de los nuevos modelos como estrategia económica, donde el mercado laboral y en especial la disparidad salarial tienen implicaciones; de esta manera son necesarios estudios sobre el comportamiento de la desigualdad salarial regional/urbano provocado por los modelos de producción implantados en México en cada región y sector económico (Castro y Huesca, 2007). Derivado del contexto antes expuesto se considera pertinente analizar los ingresos que perciben tanto mujeres como hombres en los diversos sectores de actividad económica en México, para finalmente mostrar la brecha salarial por cada actividad y determinar cuáles sectores económicos favorecen a uno u otro sexo.

El objetivo de este trabajo es analizar los ingresos de mujeres y hombres por actividad económica en México durante los últimos 15 años y a partir de esos datos detectar la brecha salarial 
mediante la descomposición Oaxaca-Blinder, finalmente establecer cuáles actividades económicas ofrecen salarios más favorables a hombres y cuáles a mujeres. La estructura de este trabajo es la siguiente, en la segunda sección se presenta un marco teórico, en el cual se abordan los conceptos de brecha salarial, estudios respecto al tema a partir de la descomposición OaxacaBlinder y el Sistema de Clasificación Industrial de América del Norte (SCIAN), en la tercera sección se presenta la metodología, aquí se describen las herramientas utilizadas para llevar a cabo el análisis, así como la selección y tratamiento de los datos; la cuarta sección describe los resultados de la investigación y se discuten los resultados encontrados, por último, la quinta sección denominada conclusiones precisa los hallazgos y cierra con el planteamiento de futuras líneas de investigación.

\section{Marco teórico}

\section{La brecha salarial de género}

Las diferencias de género han tenido su origen en la división sexual de las actividades laborales, donde a las mujeres se le han asignado las tareas relacionadas con la producción doméstica, trabajo que no es remunerado (Calderón y Campos, 2013), sin embargo, cada vez más las mujeres se incorporan de forma masiva a la fuerza de trabajo en todo el mundo. En ese sentido, la discriminación puede trasladarse al ámbito laboral pudiendo afectar el tipo de trabajo que desempeña y el salario que percibe el individuo (Braga et al., 2020).

La discriminación salarial es la parte de la diferencia que no queda justificada por una distinta aportación de valor en el desempeño de un trabajo, y que solo puede ser explicada en función del sexo de la persona que lo realiza (Díaz, 2015). Respecto a la brecha salarial de género, Sallé y Molpeceres (2010) la definen como el promedio de las retribuciones brutas por hora de las mujeres como porcentaje del promedio de las retribuciones brutas por hora de los hombres. Por su parte, Jabbaz et al. (2018) indican que mide la diferencia salarial entre mujeres y hombres en un momento y espacio determinado.

Las condiciones laborales de las mujeres unidas a la percepción más baja de salarios conforman un círculo vicioso, en el que los bajos salarios se justifican por la ocupación en categorías de bajo rango y poca presencia en puestos de dirección (García y Rendón, 2004). Si bien durante los últimos 30 años se ha acelerado la integración de las mujeres al mercado laboral y a la esfera pública (Carosio, 2012), éstas todavía enfrentan dificultades para incorporarse en 
niveles de mayor responsabilidad en los sectores público, privado, de investigación e innovación (Vizcaíno et al., 2016).

Un elemento importante en las relaciones de género es la manera en la que se solidifican las relaciones de poder en una sociedad a través de la opresión (en formas como la violencia, discriminación, marginalización) y del privilegio (Baylina, 2004). En la actualidad, existe abundante evidencia de que persisten diferencias significativas en los niveles de salarios de hombres y mujeres, comúnmente atribuidas a discriminación (Benítez y Espinoza, 2018).

Específicamente, en el ámbito laboral de México, la situación de las mujeres en relación con los ingresos las sitúa en una condición vulnerable, afectando su autonomía y empoderamiento económico (INMUJERES, 2016); siendo más frecuente la desigualdad de género en la economía informal (Valdez et al., 2021). A lo anterior se suma el hecho que la desigualdad de ingresos puede aumentar a medida que aumenta la pobreza (Ramos et al., 2020).

México reconoce los principales instrumentos internacionales que protegen los derechos de las mujeres y se alinea a los Objetivos del Desarrollo Sostenible, en particular al objetivo de Igualdad de Género. En este sentido, ha elaborado instrumentos y políticas públicas buscando erradicar la discriminación y garantizar la igualdad entre mujeres y hombres.

La discriminación en el mercado laboral es un tema reportado en la mayoría de los países del mundo, por lo tanto, son necesarias herramientas para el análisis de datos que indiquen la brecha salarial entre hombres y mujeres. En este sentido, se ha utilizado la herramienta de Oaxaca-Blinder para realizar los comparativos entre grupos, entre los que se encuentran grupos como residentes rurales-urbanos, trabajadores formales-informales y hombres-mujeres (Ñopo, 2008). Entre los estudios relacionados con el uso de la metodología Oaxaca-Blinder se puede citar a Mendoza et al. (2017), quienes reportaron que la escolaridad es un factor de discriminación en el periodo previo a 1995 y el TLCAN, dicho reporte se realizó a partir de datos de la Encuesta Nacional de Empleo Urbano (ENEU), que estuvo activa de 1987 a 2004, y la Encuesta Nacional de Ocupación y Empleo (ENOE) del INEGI.

En otro estudio realizado por Lima et al. (2021), reportaron que el salario de los hombres es superior a las mujeres aunque con una tendencia decreciente, además de se encontró discriminación por género en promedio de $17.67 \%$ de las diferencias en la remuneración entre ambos grupos. En el mismo contexto, Márquez y Reyes (2015) aplicaron la descomposición Oaxaca-Blinder para determinar la brecha de ingresos en personas con y sin discapacidad en 
ocupaciones no calificadas, encontrando desigualdad en los ingresos favorable en 1.7 veces para personas sin discapacidad. Los estudios descritos que utilizan la descomposición Oaxaca-Blinder son relevantes desde el contexto de género y brechas sociales, sin embargo las actividades económicas de México no han sido evaluadas en su conjunto para determinar la evolución de las brechas salariales entre hombres y mujeres.

\section{EI Sistema de Clasificación Industrial de América del Norte}

Para homologar la información de las actividades económicas en América del norte, la estructura económica está organizada de acuerdo al Sistema de Clasificación Industrial de América del Norte (SCIAN), sistema que proporciona un marco único, consistente y actualizado para la recopilación, análisis y presentación de estadísticas de tipo económico (INEGI, 2018). En la tabla 1 se presentan las 20 actividades económicas que conforman el SCIAN.

Tabla 1. Actividades económicas con base al SCIAN.

Table 1. Economic activities based on the SCIAN.

\begin{tabular}{|l|c|}
\hline \multicolumn{1}{|c|}{ Número y nombre } & $\begin{array}{c}\text { Abreviación } \\
\text { a utilizar }\end{array}$ \\
\hline Actividad 1. Agricultura, ganadería, aprovechamiento forestal, pesca y caza & A1 \\
\hline Actividad 2. Minería & A2 \\
\hline Actividad 3. Generación y distribución de electricidad, suministro de agua y gas & A3 \\
\hline Actividad 4. Construcción & A4 \\
\hline Actividad 5. Industrias manufactureras & A5 \\
\hline Actividad 6. Comercio al por mayor & A6 \\
\hline Actividad 7. Comercio al por menor & A7 \\
\hline Actividad 8. Transportes, correos y almacenamiento & A8 \\
\hline Actividad 9. Información en medios masivos & A9 \\
\hline Actividad 10. Servicios financieros y de seguros & A10 \\
\hline Actividad 11. Servicios inmobiliarios y de alquiler de bienes & A11 \\
\hline Actividad 12. Servicios profesionales, científicos y técnicos & A12 \\
\hline Actividad 13. Corporativos & A13 \\
\hline Actividad 14. Servicios de apoyo a los negocios y manejo de desechos & A14 \\
\hline Actividad 15. Servicios educativos & A15 \\
\hline Actividad 16. Servicios de salud y asistencia social & A16 \\
\hline Actividad 17. Servicios de esparcimiento culturales y deportivos & A17 \\
\hline Actividad 18. Servicios de hospedaje y preparación de alimentos y bebidas & A18 \\
\hline Actividad 19. Otros servicios, excepto actividades gubernamentales & A19 \\
\hline Actividad 20. Actividades gubernamentales y de organismos internacionales & A20 \\
\hline
\end{tabular}

Fuente: elaboración propia a partir de la ENOE Estructura de la base de datos, 2019. Source: own elaboration based on ENOE Structure of the database, 2019. 


\section{Método}

Los datos para realizar este análisis se obtuvieron de la Encuesta Nacional de Ocupación y Empleo, la cual es una encuesta realizada por el Instituto Nacional de Estadística y Geografía (INEGI) que ofrece datos tanto mensuales como trimestrales y representa la principal fuente de información sobre el mercado laboral mexicano, cuenta con datos históricos de 2005 a la actualidad de cada una de las 32 entidades federativas (INEGI, 2021). De la encuesta antes mencionada se descargó la información correspondiente al tercer trimestre de cada año de 2005 al 2019; se obtuvo información sobre ingresos por hora, años de escolaridad y actividad económica, ambos datos tanto para hombres y mujeres con edades de 15 a 59 años y con nivel educativo de primaria a posgrado.

Los ingresos por hora que se seleccionaron anteriormente fueron deflactados a partir de la fórmula 1. El valor de la inflación que señala la fórmula 1 se tomó del índice de precios al consumidor del año 2019 emitido por el Banco de México:

Ingresos por hora deflactados $=\frac{\text { Ingresos por hora del periodo actual }}{\text { Valor de la inflación del periodo actual }} *$ Valor de la inflación del periodo de referencia

En seguida se obtuvieron los ingresos promedio de hombres y mujeres por año de análisis y por actividad económica, así mismo se calculó la experiencia mediante la fórmula 2 (Urciaga y Almendarez, 2006). Notar que para calcular este dato se ocuparon los valores de edad y años de escolaridad que se extrajeron de la ENOE.

$$
\text { Experiencia }=\text { edad }- \text { años de escolaridad }-6
$$

Una vez completa la información requerida, se elaboró un análisis econométrico mediante mínimos cuadrados ordinarios a partir de la función de ingresos de Mincer que se muestra en la fórmula 3 (Bunzel, 2008) :

$$
\ln Y=\beta_{0}+\beta_{1} S+\beta_{2} t+\beta_{3} t^{2}+\varepsilon
$$


Donde:

$\ln Y$ corresponde al logaritmo natural de los ingresos,

$\mathrm{S}$ corresponde al número de años de escolaridad,

t corresponde al número de años de experiencia,

$t^{2}$ corresponde a la experiencia ${ }^{2}, \mathrm{y}$

$\varepsilon$ es el error estadístico no correlacionado con $\mathrm{S}$ y t.

Notar que al realizar las regresiones correspondientes, se decidió no contemplar la experiencia ${ }^{2}$ con la intención de evitar multicolinealidad, quedando la función de ingreso antes mencionada, de la siguiente manera:

$$
\ln Y=\beta_{0}+\beta_{1} S+\beta_{2} t+\varepsilon
$$

Finalmente se identificaron las diferencias salariales entre mujeres y hombres en las actividades económicas a través del método de descomposición Oaxaca-Blinder (1973), cuya ecuación básica de descomposición es:

$$
R=\left(\bar{X}_{A}-\bar{X}_{B}\right) \hat{\beta}_{A}+\bar{X}_{B}\left(\bar{\beta}_{A}-\bar{\beta}_{B}\right)
$$

Donde:

A corresponde al grupo de hombres;

B corresponde al grupo de mujeres;

$\bar{\beta}$ es la $\beta_{1}$ obtenida en la función de salarios de Mincer para cada grupo;

$\bar{X}$ representa los ingresos medios deflactados para cada grupo.

En la fórmula 5 se observa que la diferencia en los salarios de hombres y mujeres es la suma de dos componentes, el primero de ellos identificado como la parte explicada por las diferencias entre las variables observadas y el segundo como la parte no explicada o asociada a las variables no observadas (Vicéns, 2012). 


\section{$\underline{\text { Resultados }}$}

El número total de individuos que conformaron el presente análisis fue 2066 711, de los cuales el $40.14 \%$ son mujeres, y el $59.86 \%$ son hombres; en la tabla 2 se muestra la información desglosada por sexo y por cada actividad económica. En dicha tabla se observan aquellas actividades económicas en las cuales predomina la participación de los hombres; destacan las Actividades de Construcción (A4); Transportes, correos y almacenamiento (A8); Agricultura, ganadería, aprovechamiento forestal, pesca y caza (A1); y Minería (A2).

Por otra parte, destacan las actividades de Servicios de salud y asistencia social (A16); y Servicios educativos (A15) con una mayor participación de las mujeres. Merece especial atención la actividad de Servicios Financieros y de Seguros (A10), pues es la actividad en la que la participación de hombres y mujeres se acerca más a ser equivalente: participa $48.76 \%$ hombres y $51.24 \%$ mujeres, se tiene un $2.49 \%$ de diferencia.

Tabla 2. Individuos que conformaron el análisis por actividad económica y sexo. En cantidad y porcentaje.

Table 2. Individuals that make up the analysis by economic activity and sex. In quantity and percentage.

\begin{tabular}{|c|c|c|c|c|c|}
\hline $\begin{array}{l}\text { Actividad } \\
\text { Económica }\end{array}$ & $\begin{array}{l}\text { Número de } \\
\text { individuos por sexo }\end{array}$ & $\begin{array}{l}\text { Porcentaje que } \\
\text { representa cada } \\
\text { sexo }\end{array}$ & $\begin{array}{l}\text { Actividad } \\
\text { Económica }\end{array}$ & $\begin{array}{l}\text { Número de } \\
\text { individuos por } \\
\text { sexo }\end{array}$ & $\begin{array}{l}\text { Porcentaje que } \\
\text { representa cada } \\
\text { sexo }\end{array}$ \\
\hline A1 & $\begin{array}{l}\mathrm{H}=123851 \\
\mathrm{M}=15396\end{array}$ & $\begin{array}{l}\mathrm{H}=88.94 \% \\
\mathrm{M}=11.06 \%\end{array}$ & A11 & $\begin{array}{l}H=9176 \\
M=5272\end{array}$ & $\begin{array}{l}\mathrm{H}=63.51 \% \\
\mathrm{M}=36.49 \%\end{array}$ \\
\hline A2 & $\begin{array}{l}\mathrm{H}=10523 \\
\mathrm{M}=1503\end{array}$ & $\begin{array}{l}\mathrm{H}=87.50 \% \\
\mathrm{M}=12.50 \%\end{array}$ & A12 & $\begin{array}{l}\mathrm{H}=34082 \\
\mathrm{M}=23059\end{array}$ & $\begin{array}{l}\mathrm{H}=64.55 \% \\
\mathrm{M}=40.35 \%\end{array}$ \\
\hline A3 & $\begin{array}{l}\mathrm{H}=8584 \\
\mathrm{M}=2328\end{array}$ & $\begin{array}{l}\mathrm{H}=78.67 \% \\
\mathrm{M}=21.33 \%\end{array}$ & A13 & $\begin{array}{c}\mathrm{H}=1026 \\
\mathrm{M}=858\end{array}$ & $\begin{array}{l}\mathrm{H}=54.46 \% \\
\mathrm{M}=45.54 \%\end{array}$ \\
\hline A4 & $\begin{array}{c}\mathrm{H}=152640 \\
\mathrm{M}=7735\end{array}$ & $\begin{array}{l}\mathrm{H}=95.18 \% \\
\mathrm{M}=4.82 \%\end{array}$ & A14 & $\begin{array}{l}\mathrm{H}=35635 \\
\mathrm{M}=20137\end{array}$ & $\begin{array}{l}\mathrm{H}=63.89 \% \\
\mathrm{M}=39.12 \%\end{array}$ \\
\hline A5 & $\begin{array}{l}\mathrm{H}=219752 \\
\mathrm{M}=122861\end{array}$ & $\begin{array}{l}\mathrm{H}=64.14 \% \\
\mathrm{M}=35.86 \%\end{array}$ & A15 & $\begin{array}{l}\mathrm{H}=53436 \\
\mathrm{M}=87242\end{array}$ & $\begin{array}{l}\mathrm{H}=37.98 \% \\
\mathrm{M}=62.02 \%\end{array}$ \\
\hline A6 & $\begin{array}{l}\mathrm{H}=46694 \\
\mathrm{M}=16942\end{array}$ & $\begin{array}{l}\mathrm{H}=73.38 \% \\
\mathrm{M}=26.62 \%\end{array}$ & A16 & $\begin{array}{l}\mathrm{H}=25904 \\
\mathrm{M}=56307\end{array}$ & $\begin{array}{l}\mathrm{H}=31.51 \% \\
\mathrm{M}=68.49 \%\end{array}$ \\
\hline A7 & $\begin{array}{l}H=159222 \\
M=194156\end{array}$ & $\begin{array}{l}\mathrm{H}=45.06 \% \\
\mathrm{M}=54.94 \%\end{array}$ & A17 & $\begin{array}{c}\mathrm{H}=14857 \\
\mathrm{M}=5810\end{array}$ & $\begin{array}{l}\mathrm{H}=71.89 \% \\
\mathrm{M}=28.11 \%\end{array}$ \\
\hline A8 & $\begin{array}{l}\mathrm{H}=85781 \\
\mathrm{M}=8307\end{array}$ & $\begin{array}{l}\mathrm{H}=91.17 \% \\
\mathrm{M}=8.83 \%\end{array}$ & A18 & $\begin{array}{l}\mathrm{H}=65594 \\
\mathrm{M}=81566\end{array}$ & $\begin{array}{l}\mathrm{H}=44.57 \% \\
\mathrm{M}=55.43 \%\end{array}$ \\
\hline A9 & $\begin{array}{c}\mathrm{H}=12607 \\
\mathrm{M}=7184\end{array}$ & $\begin{array}{l}\mathrm{H}=63.70 \% \\
\mathrm{M}=36.30 \%\end{array}$ & A19 & $\begin{array}{c}H=86654 \\
M=105169\end{array}$ & $\begin{array}{l}\mathrm{H}=45.17 \% \\
\mathrm{M}=54.83 \%\end{array}$ \\
\hline A10 & $\begin{array}{l}\mathrm{H}=11299 \\
\mathrm{M}=11875\end{array}$ & $\begin{array}{l}\mathrm{H}=48.76 \% \\
\mathrm{M}=51.24 \%\end{array}$ & A20 & $\begin{array}{l}\mathrm{H}=79763 \\
\mathrm{M}=55924\end{array}$ & $\begin{array}{l}\mathrm{H}=58.78 \% \\
\mathrm{M}=41.22 \%\end{array}$ \\
\hline
\end{tabular}

Fuente: elaboración propia a partir de los datos de la ENOE 2005 a 2019, 3er. Trimestre.

Abreviaciones: $\mathrm{H}=$ Hombres, $\mathrm{M}=$ Mujeres, para A1 hasta A20 (ver tabla 1).

Source: own elaboration based on data from ENOE 2005 to 2019, 3rd. Trimester.

Abbreviations: $\mathrm{H}=\mathrm{Men}, \mathrm{M}=$ Women, for A1 to A20 (see table 1). 
Para cada año de análisis, actividad económica y sexo se realizó una regresión múltiple, utilizando la función de ingresos de Mincer sin la experiencia ${ }^{2}$ (ver fórmula 4). En tal regresión, la variable explicada fue la de los ingresos por hora, y como variables explicativas se tuvieron los años de escolaridad y experiencia. En la tabla 3 se ejemplifican los resultados de este paso para la actividad económica de Servicios Financieros y de Seguros (A10).

Tabla 3. Resultados de la regresión múltiple por sexo y año. Actividad económica: Servicios financieros y de seguros (A10).

Table 3. Results of the multiple regression by sex and year. Economic activity: Financial and insurance services (A10).

\begin{tabular}{|c|c|c|c|c|c|c|c|c|}
\hline \multirow{2}{*}{ Año } & \multicolumn{4}{|c|}{ Hombres } & \multicolumn{4}{c|}{ Mujeres } \\
\cline { 2 - 9 } & $\beta_{0}$ & $\begin{array}{c}\beta_{1}, \text { Años de } \\
\text { escolaridad }\end{array}$ & $\begin{array}{c}\beta_{2}, \\
\text { Experiencia }\end{array}$ & $R^{2}$ & $\beta_{0}$ & $\begin{array}{c}\beta_{1} \text {, Años de } \\
\text { escolaridad }\end{array}$ & $\beta_{2}$, Experiencia & $R^{2}$ \\
\hline 2005 & 2.5919 & 0.0964 & 0.0152 & 16.4985 & 2.1151 & 0.1101 & 0.0248 & 24.4133 \\
\hline 2006 & 2.3153 & 0.1066 & 0.0205 & 22.0480 & 2.1354 & 0.1084 & 0.0268 & 26.2666 \\
\hline 2007 & 2.3999 & 0.1073 & 0.0162 & 22.2968 & 2.3988 & 0.0972 & 0.0188 & 18.4989 \\
\hline 2008 & 2.5637 & 0.0822 & 0.0257 & 19.6954 & 2.3837 & 0.0927 & 0.0185 & 21.7643 \\
\hline 2009 & 2.3676 & 0.0928 & 0.0227 & 21.5134 & 2.5600 & 0.0809 & 0.0124 & 13.5786 \\
\hline 2010 & 2.3444 & 0.0990 & 0.0159 & 18.4958 & 2.4424 & 0.0852 & 0.0199 & 14.7300 \\
\hline 2011 & 2.3042 & 0.0977 & 0.0186 & 23.5723 & 2.2321 & 0.0907 & 0.0218 & 16.7110 \\
\hline 2012 & 2.0467 & 0.1124 & 0.0186 & 25.6854 & 2.5500 & 0.0772 & 0.0117 & 11.1699 \\
\hline 2013 & 2.5782 & 0.0820 & 0.0109 & 12.0637 & 2.2750 & 0.0876 & 0.0217 & 14.5906 \\
\hline 2014 & 1.9201 & 0.1156 & 0.0227 & 25.4804 & 2.3406 & 0.0829 & 0.0172 & 14.9285 \\
\hline 2015 & 2.2019 & 0.0995 & 0.0165 & 17.2658 & 2.1471 & 0.0985 & 0.0193 & 18.3357 \\
\hline 2016 & 2.1642 & 0.1005 & 0.0170 & 18.3149 & 2.6327 & 0.0688 & 0.0129 & 9.5452 \\
\hline 2017 & 2.6710 & 0.0710 & 0.0080 & 9.7298 & 2.5250 & 0.0692 & 0.0171 & 11.1562 \\
\hline 2018 & 2.5106 & 0.0781 & 0.0127 & 14.2258 & 2.2083 & 0.0927 & 0.0164 & 17.5650 \\
\hline 2019 & 2.2477 & 0.0981 & 0.0166 & 17.0517 & 2.2350 & 0.0933 & 0.0170 & 15.6518 \\
\hline
\end{tabular}

Fuente: elaboración propia.

Source: own elaboration.

Los datos anteriores sirvieron para obtener la diferencia salarial entre mujeres y hombres a partir de la descomposición Oaxaca-Blinder (fórmula 5); los valores positivos representan una brecha salarial favorable de los hombres y los valores negativos representan una brecha salarial favorable a las mujeres. En la tabla 4, se observa que las actividades de Construcción (A4) y Transportes, correos y almacenamiento (A8) durante los 15 años de estudio han mostrado salarios favorables a las mujeres. En ambas actividades predomina la participación de los hombres y, por tanto, la existencia de una baja participación de las mujeres, en razón de 19.73 hombres por cada mujer en la Construcción y 10.32 hombres por cada mujer en Transportes, correos y almacenamiento.

Por el contrario, las actividades Corporativos (A13) y Otros servicios, excepto actividades gubernamentales (A19) han mostrado históricamente salarios favorables a los hombres, en lo que 
respecta a la incorporación de hombres y mujeres en estas actividades es de 1.19 y 0.82 hombres por cada mujer, respectivamente. Algunas otras actividades presentan un histórico favorable a los hombres, pero ya empieza aparecer al menos un año favorable a las mujeres, como lo es el caso de las actividades de Servicios profesionales, científicos y técnicos (A12), Servicios educativos (A15) y Servicios de hospedaje y preparación de alimentos y bebidas (A18).

La actividad de Servicios de Salud y Asistencia Social (A16) es donde mayor participación hay de mujeres respecto a hombres, en razón de 0.46 hombres por cada mujer; sin embargo, el salario ha sido favorable a los hombres en la mayoría de los años, siendo la excepción los años 2011 y 2014. Respecto a la actividad de Servicios Financieros, donde la participación de hombres y mujeres es la más equitativa en cuanto a cantidad, de 2005 a 2016 y 2019 los salarios han sido favorables a los hombres, solamente en 2017 y 2018 favorecieron a las mujeres.

Tabla 4. Resultados de la descomposición Oaxaca-Blinder por año y actividad económica.

Table 4. Results of the Oaxaca-Blinder decomposition by year and economic activity.

\begin{tabular}{|c|c|c|c|c|c|c|c|c|c|c|c|c|c|c|c|c|c|c|c|c|}
\hline 1 & $\frac{\pi}{4}$ & 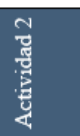 & 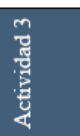 & 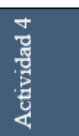 & 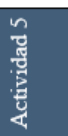 & 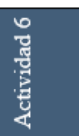 & 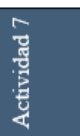 & $\begin{array}{l}\infty \\
0 \\
0 \\
0 \\
0 \\
0 \\
0\end{array}$ & 造 & 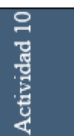 & 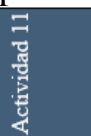 & $\begin{array}{l}\text { 궁 } \\
\text { g] } \\
\frac{\pi}{3} \\
\frac{3}{4}\end{array}$ & 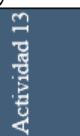 & 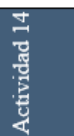 & $\frac{\tilde{}}{g}$ & $\begin{array}{l}0 \\
0 \\
0 \\
\frac{\pi}{0} \\
0 \\
0 \\
0\end{array}$ & $\begin{array}{l}7 \\
\overline{0} \\
\frac{\pi}{3} \\
\frac{\pi}{4}\end{array}$ & 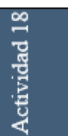 & $\begin{array}{l}9 \\
\frac{9}{7} \\
\frac{\pi}{7} \\
\frac{3}{0} \\
\frac{\pi}{4}\end{array}$ & 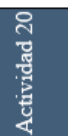 \\
\hline 2005 & 018 & 1.560 & 340 & 162 & 032 & $\overline{119}$ & 268 & 747 & 802 & 97 & 36 & 417 & 90 & .034 & 638 & 149 & 342 & 457 & .977 & 0.011 \\
\hline $200 c$ & 575 & 966 & 128 & 512 & 628 & .362 & 342 & .445 & 609 & 893 & 26 & .798 & 673 & 514 & .745 & .149 & 504 & 829 & .833 & 129 \\
\hline 20 & 332 & 2.068 & 18 & 734 & 679 & 060 & 96 & 584 & 000 & 1.657 & 347 & 42 & 395 & 58 & 99 & 53 & 030 & 77 & 030 & 118 \\
\hline $200 \mathrm{~s}$ & 331 & 2.269 & 160 & 958 & 332 & 0.099 & 396 & 852 & 19 & 0.633 & 1.564 & $6 / 7$ & 997 & 0.108 & 77 & .0942 & 3.626 & 40 & 679 & .367 \\
\hline 200 & 476 & 1.129 & 146 & .908 & 401 & 113 & 166 & .27 & .591 & .35 & 2.003 & 1.015 & 3.338 & 66 & 0.013 & 1.089 & 0.665 & 93 & 4.438 & 0.128 \\
\hline 2010 & 492 & -1.528 & 809 & 283 & 300 & 327 & 157 & 2.156 & .115 & 616 & 0.184 & .036 & 7.895 & 0.380 & -0.125 & 1.054 & 0.266 & 451 & 0.876 & .156 \\
\hline 2011 & .007 & 148 & 243 & 841 & .279 & 142 & .124 & .245 & 1.027 & 117 & -5.656 & 1.151 & 4.119 & -0.424 & 0.612 & 0.037 & -2.324 & .204 & 0.550 & -0.144 \\
\hline 2012 & 0.051 & 0.065 & 0.517 & 877 & 273 & 350 & .064 & .772 & .315 & 05 & 5.190 & 0.302 & 2.703 & 0.321 & 0.701 & 0.003 & 1.014 & 342 & .755 & 0.038 \\
\hline 2013 & .271 & 397 & 0.869 & .028 & .033 & 0.418 & .021 & 662 & .636 & 0.026 & 2.622 & 399 & 3.192 & 87 & 96 & 0.252 & 58 & 71 & 0.596 & .030 \\
\hline 2014 & -0.187 & -0.439 & 0.609 & -2.024 & 0.100 & 0.355 & 0.100 & -1.428 & 0.605 & 1.583 & 713 & 0.833 & 0.113 & -0.019 & 0.618 & -0.351 & 0.069 & 376 & 0.441 & .024 \\
\hline 2015 & -0.372 & -1.734 & 0.538 & 1.077 & 228 & .570 & -0.081 & -1.421 & 0.500 & 0.368 & 2.153 & 0.402 & 14.553 & 0.237 & 0.209 & 0.857 & -1.783 & .163 & 0.800 & 0.390 \\
\hline 2016 & 0.160 & 0.541 & -0.250 & -0.968 & 0.300 & -0.215 & 0.059 & -2.451 & -0.084 & 1.408 & 1.192 & 0.800 & 0.863 & 0.036 & 4.436 & .353 & -1.974 & 0.255 & 0.552 & 0.269 \\
\hline 2017 & -0.413 & -3.153 & 0.254 & -1.059 & .124 & 0.145 & -0.092 & -1.804 & 0.957 & 0.016 & 1.242 & 0.608 & 1.463 & -0.299 & .162 & .627 & -1.375 & 0.004 & 0.265 & 0.539 \\
\hline 2018 & -0.143 & -1.028 & 185 & .184 & .154 & 0.069 & 0.067 & -1.542 & -0.509 & 0.323 & -2.704 & 0.714 & 271 & -0.162 & .256 & .109 & -0.447 & 275 & 0.584 & 162 \\
\hline & & -2.677 & & 18 & & 21 & 022 & 041 & & & & & 8.851 & 0.442 & 401 & 176 & 86 & 71 & 324 & 372 \\
\hline & 003 & .666 & 344 & .153 & 302 & .078 & 059 & .803 & 689 & .813 & 0.952 & .687 & .434 & .069 & .449 & .813 & 956 & 371 & .632 & 073 \\
\hline
\end{tabular}

Fuente: elaboración propia a partir de los datos de la ENOE de los años 2005 al 2019, 3er. Trimestre.

Source: Own elaboration based on ENOE data from 2005 to 2019, 3rd. Trimester.

Una causa observada de la brecha salarial es la distribución del empleo por ocupación, las actividades de construcción, así como transportes, correos y almacenamiento. En los años de análisis presentan una brecha salarial favorable a las mujeres y coincide con que la participación de la mujer en estos sectores es baja con respecto a la participación de los hombre. Bustelo y Vezza (2019) argumentan que mientras casi un $30 \%$ de mujeres trabajan en sectores vinculados al cuidado, solo se tiene un $6 \%$ de hombres en el mismo sector, en ese sentido, observamos que del 
$100 \%$ de las personas que conformaron este estudio y que se ocupan en la actividad económica de la construcción, solo el $4.82 \%$ son mujeres. Por su parte, en lo que respecta a la actividad económica de transportes, correos y almacenamientos solamente, el $8.83 \%$ son mujeres.

Las actividades 13-Corporativos y 19-Otros servicios, excepto actividades gubernamentales, han mostrado históricamente salarios favorables a los hombres. La participación de la mujer en estas actividades es importante: del $100 \%$ de las personas que se analizaron de dichas actividades económicas, el $45.54 \%$ son mujeres en la actividad de corporativos, y el 54.83 $\%$ son mujeres en la actividad de otros servicios, excepto actividades gubernamentales. Específicamente, las mujeres que laboran en estas actividades económicas se encuentran en una condición económicamente vulnerable. Rodríguez-Pérez et al. (2017) describieron que la brecha salarial por género en México era favorable a las mujeres en el sector público y desfavorable en el sector privado, específicamente en el sector informal.

En la fig. 1 se presenta la cantidad de actividades económicas que favorecen a hombres y mujeres, respectivamente. Los resultados muestran un avance en la disminución de la desigualdad salarial; se observa cada vez mayor número de actividades económicas con salarios favorables a las mujeres. En particular, en el año 2005, 15 actividades económicas favorecían económicamente a los hombres, frente a 5 que favorecían a las mujeres. Para el año 2019, son 9 las actividades económicas que favorecen a los hombres frente a 11 a favor de las mujeres.

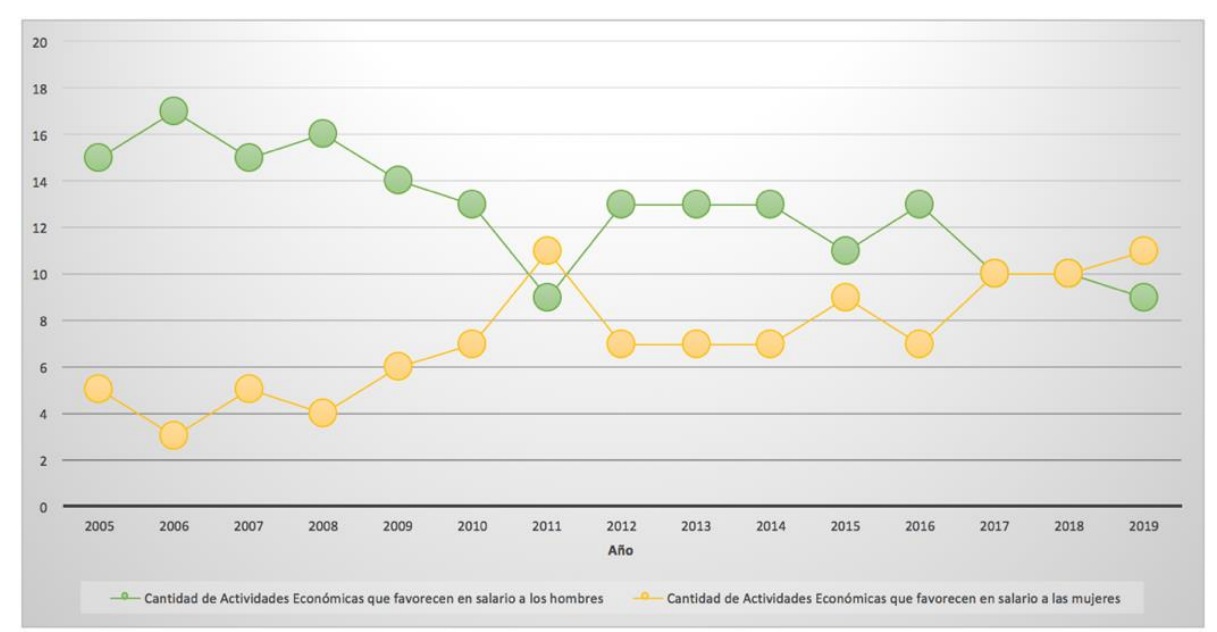

Fig. 1. Número de actividades económicas favorables en salario a hombres y mujeres.

Fig 1. Number of economic activities favorable in salary to men and women. 


\section{Discusión o conclusiones}

Este trabajo de investigación comenzó exponiendo la situación de desigualdad a la que se enfrentan las mujeres en diversos ámbitos de la vida, no siendo la excepción el entorno laboral; se explicó además de la necesidad de desagregar por actividad económica las brechas salariales. Se presentó un análisis de los ingresos a partir de la función de salarios de Mincer y de la descomposición Oaxaca-Blinder. La función de ingresos de Mincer ayudó a estimar la relación entre la escolaridad y la experiencia con los ingresos de hombres y mujeres en las distintas actividades económicas. Luego, la descomposición Oaxaca-Blinder permitió obtener la diferencia salarial, de la cual una parte se explica a través de las características observables como la educación y la experiencia.

Los resultados obtenidos plantean la existencia de una brecha salarial, históricamente favorable en algunas actividades económicas a los hombres, por ejemplo, las actividades de Corporativos y Otros servicios, excepto actividades gubernamentales. Por el contrario, en las actividades de Construcción y Transportes, así como Correos y Almacenamiento, la brecha salarial ha sido históricamente favorable a las mujeres; cabe destacar que en estas actividades las mujeres tienen menor presencia en razón del número de hombres que ahí laboran. Se observó que las actividades económicas en las cuales la participación de un sexo (hombre o mujer) es baja con respecto al otro, ofrecen ingresos superiores para el sexo menor representado (ver los resultados para las actividades 4,8 y 16$)$.

A lo largo del periodo de análisis, conformado por 15 años, se observa un crecimiento en cuanto al número de actividades económicas que ofrecen mejores ingresos económicos a las mujeres. En el año 2005, 5 de 20 actividades económicas ofrecían ingresos favorables a las mujeres, mientras que 15 de las 20 actividades lo hacía para los hombres; para el año 2019 se tuvieron 11 actividades con mejores ingresos para las mujeres, en comparación con las 9 actividades con mejores ingresos para los hombres. Cabe señalar que si esa tendencia continúa, cada vez más actividades económicas favorecerán en ingresos a las mujeres, lo que podría provocar que ahora sean los hombres quienes estén en una situación de vulnerabilidad y discriminación.

Con los resultados alcanzados se dio respuesta a los planteamientos inicialmente hechos en este trabajo; se analizaron los ingresos por sexo y actividad económica en México a lo largo de un periodo de 15 años, y se detectó la existencia de una brecha salarial por actividad económica, pudiendo establecer aquellas favorables para uno u otro sexo. La evidencia de la brecha salarial por género se expone a partir de las variables explicativas observables (los años de escolaridad, 
experiencia e ingresos por hora), quedando limitada a aquellas variables no observadas directamente en los datos utilizados. En general, a pesar de observarse una disminución en la brecha salarial durante el periodo de análisis, las desigualdades en los ingresos persisten entre hombres y mujeres en las distintas actividades económicas.

Con este trabajo, es claro que las estrategias y políticas públicas que se implementen en favor de una igualdad salarial deben diseñarse a la medida en función de las necesidades y características particulares de cada actividad. Cada una de las actividades económicas tiene un comportamiento distinto en cuanto a la participación de hombres y mujeres, así como en cuanto a los ingresos que ambos sexos perciben.

Es importante indicar que el análisis de la brecha salarial aquí mostrado se limitó a las variables planteadas de años de escolaridad, experiencia e ingresos por hora; por lo tanto se recomienda ampliar este tipo de análisis a otras variables.

Para plantear la ampliación de estos análisis, una pregunta interesante podría ser: ¿Qué otros factores están asociados con una menor o mayor remuneración hacia la mujer con respecto a los hombres en cada actividad económica? Además, se podría puntualizar este mismo estudio incorporando resultados por nivel educativo y entidad federativa.

\section{Agradecimientos}

La primer autora agradece al Consejo Nacional de Ciencia y Tecnología (CONACYT) por la beca otorgada para la realización de sus estudios de Doctorado.

\section{Referencias}

Abramo, L. (2004). ¿Inserción laboral de las mujeres en América Latina: una fuerza de trabajo secundaria? Estudios feministas, Florianópolis, 12(2), 224-235. https://www.redalyc.org/articulo.oa?id=38112213

Baylina, M., Villarino, M., García Ramos, M. D., Mosteiro, M. J., Porto A. M. y Salamaña, I. (2019). Género e innovación en los nuevos procesos de re-ruralización en España. Finisterra, LIV(110), 75-91. DOI: 10.18055/finis16053

Benítez, D. y Espinoza, B. (2018). Discriminación salarial por género en el sector formal en Ecuador usando registros administrativos. Ecuador: Instituto Nacional de Estadística y Censos. 
https://www.ecuadorencifras.gob.ec/documentos/webinec/Bibliotecas/Libros/Discriminac ion_salar_por_genero_sec_for_Ecu.pdf

Braga, D. de L., Cuenca, A. D., y Da Cunha, F. C. (2020). Discriminación salarial por género en el mercado de trabajo del Paraguay: Análisis del sector formal, Zona Metropolitana y Zona Fronteriza con Brasil. Estudios económicos, 38(76), 5-43. https://www.redalyc.org/jatsRepo/5723/572365672001/html/

Bucio, A. (2014). Brechas de género: La mujer mexicana entre el suelo pegajoso y el techo de cristal. [Tesis de Licenciatura. Facultad de Ciencias Políticas del Instituto Tecnológico Autónomo de México]. Ciudad de México.

Bunzel, H. (2008). Mincer Model. Disponible en: http://www2.econ.iastate.edu/classes/econ671/bunzel/Fall2008/Mincerbeam.pdf

Bustelo M. y Vezza, E. (2019). Brechas de género en las habilidades para el siglo XXI. En Mateo Díaz, M. y Rucci, G. El futuro ya está aquí: Habilidades transversales en América Latina y el Caribe en el Siglo XXI (pp. 82-106). Banco Interamericano de Desarrollo.

Cadena, M. Y. (2020). Discriminación salarial por género: análisis de las empresas del sector privado en el eje central de Bolivia. Investigación y desarrollo, 20(2), 25-40. http://www.scielo.org.bo/scielo.php?pid=S251844312020000200002\&script=sci_abstract

Cardona, R. G. A., Cardenete, F. M. A. y Martínez, G. C. I. (2018). Mexican economic structure: key, strategic, drivers and independent sectors 2012. Revista de Economía, 35, 9-50.

Carosio, A. (2012). Feminismo y cambio social en América Latina y el Caribe. Buenos Aires: CLACSO.

Calderón, Y. P. y Campos, R. G. (2013). Las mujeres jóvenes de México: ¿Estudian o trabajan? Última Década, 21, 63-83. DOI: 10.4067/S0718-22362013000200004

Castro, L. D. y Huesca, R. L. (2007). Desigualdad salarial en México: una revisión. Papeles de población, 54, 225-264.

http://www.scielo.org.mx/scielo.php?pid=S140574252007000400009\&script=sci_arttext

De la Torre, H. C., Cuamea, D. I. y Olivas, E. (2020). Incorporación de mujeres al mercado laboral y su distribución espacial por áreas de marginación en Hermosillo, Sonora, México. Géneros, 27(28), 161-194. 
Díaz, E. (2015). La desigualdad salarial entre hombres y mujeres: alcances y limitaciones en la ley No. 20.348 para avanzar en la justicia de género. Cuaderno de investigación, 55, Santiago de Chile.

Flores, P. L. y Salas, D. I. A. (2015). Las brechas de género en la calidad del empleo en México. Una valoración basada en modelos de lógica difusa. Análisis Económico, 30, 89-112. https://www.redalyc.org/pdf/413/41343702004.pdf

García, S. C. y Rendón, G. T. (2004). Female employment in México and Spain: A comparative analysis of current features. Revista de Economía Mundial, 10/11, 23-57. http://www.semwes.org/sites/default/files/revistas/rem10-11_2_0.pdf

INEGI, (2020). Censo de Población y Vivienda 2020. Disponible en: https://www.inegi.org.mx/programas/ccpv/2020/

INEGI, (2018). Síntesis metodológica del Sistema de Clasificación Industrial de América del Norte, México SCIAN 2018. Disponible en: https://www.inegi.org.mx/app/scian/

INMUJERES. (2016). Brecha salarial de género en México. Disponible en: http://cedoc.inmujeres.gob.mx/documentos_download/101271.pdf

Jabbaz, M., Samper - Gras, T. y Díaz, C. (2018). La brecha salarial de género en las instituciones científicas. Estudio de caso. Convergencia, revista de ciencias sociales, 80, 1-27. DOI: $10.29101 /$ crcs.v26i80.11248

Martínez, J. I. y Acevedo, F. C. J. (2004). La brecha salarial en México con enfoque de género: Capital humano, discriminación y selección muestral. Ciencia UANL, 7, 66-71. http://eprints.uanl.mx/1521/

Márquez, C. y Reyes, J. (2015). Análisis de la brecha de ingresos entre las personas con y sin discapacidad en ocupaciones no calificadas. Revista Latinoamericana de Población, 9(16), 1-24. https://www.redalyc.org/pdf/3238/323841354003.pdf

Mendoza González, M. A., Cardero García, M. E. y Ortíz García, A. S. (2017). Algunos hechos estilizados y explicativos sobre el diferencial y la discriminación salarial por sexo en México, 1987-2015. Investigación Económica. LXXVI(30), 103-135. http://www.scielo.org.mx/scielo.php?pid=S018516672017000300103\&script=sci_abstra $\underline{\mathrm{ct} \& \mathrm{tlng}=\mathrm{pt}}$

Moctezuma, N. D., Narro, R. J. y Orozco, H. L. (2014). Women in Mexico: Inequality, poverty, and violence. Revista Mexicana de Ciencias Políticas y Sociales, 220, 117-146. 
http://www.scielo.org.mx/scielo.php?pid=S018519182014000100005\&script=sci_abstra ct\&tlng=en

Ñopo, H. (2008). An extension of the Blinder-Oaxaca decomposition to a continuum of comparison groups. Economics Letters, 100(2), 292-296. DOI:10.1016/j.econlet.2008.02.011

Pedrero, M. (2004). Género, trabajo doméstico y extradoméstico en México. Una estimación del valor económico del trabajo doméstico. Estudios demográficos y urbanos, 19(2), 413-446. https://www.jstor.org/stable/40315187

Ramos, M. E., Gibaja-Romero, D. E. y Ochoa, S. A. (2020). Gender inequality and gender-based poverty in Mexico. Heliyon, 6, 1-10. DOI: 10.1016/j.heliyon.2020.e03322

Rodríguez-Pérez, R., Ramos-Lobo, R. y Castro-Lugo, D. (2017). Brecha salarial por género en los mercados de trabajo público y privado en México. Panorama Económico, 25, 149-172. https://repositorio.unicartagena.edu.co/handle/11227/7972

Saavedra, M. L. y Camarena, M. E. (2020). Las brechas de género y el empoderamiento femenino en México. Géneros, 27(28), 219-246. https://www.researchgate.net/profile/Maria-Luisa$\underline{\text { SaavedraGarcia/publication/354204217_Brechas_de_genero_y_empoderamiento/links/6 }}$ 12bdca70360302a0066fd94/Brechas-de-genero-y-empoderamiento.pdf

Sallé, M. A. y Molpeceres, L. (2010). La brecha salarial: realidades y desafíos. Las desigualdades salariales entre mujeres y hombres España 2009. España: Ministerio de igualdad, colección economía, mujer, empresa.

Sosa, M. M. V. y Román, R. R. P. (2015). Participation and distribution of time in daily activities of men and women working in the labor market in Mexico. Revista Sociedad y Economía, 29, 63-89.

http://www.scielo.org.co/scielo.php?script=sci_arttext\&pid=S1657-63572015000200004

Urciaga, J. y Almendarez, M. A. (2006). Determinación de los salarios y rendimientos de la escolaridad en la región Mar de Cortés. Revista de la educación superior, 35(2), 37 - 53. https://www.redalyc.org/pdf/604/60413802.pdf

Valdez, Z., Pérez, Y., Lavoignet, B. J., Cruz, F. y Hernández, C. (2021). Acontecimientos vitales estresantes en mujeres trabajadoras de un mercado del estado de Veracruz, México. Revista Colombiana de Salud Ocupacional, 11(1), 1-6. DOI: 10.18041/2322634X/rcso.1.2021.6536 
Vicéns, J. (2012). Descomposición Oaxaca-Blinder en modelos lineales y no lineales. Madrid: Instituto L. R. Klein, Universidad Autónoma de Madrid. Disponible en: https://docplayer.es/56307450-Descomposicion-oaxaca-blinder-en-modelos-lineales-yno-lineales.html

Vizcaino-Suárez, L. P. y Díaz-Carrión, I. A. (2018). Gender in Tourism Research: Perspectives from Latin America. Tourism Review. DOI: https://doi.org/10.1108/TR-02-2017-0021

Zabludovsky, G. (2007). Las mujeres en México: Trabajo, educación superior y esferas de poder. Políticas y cultura, 28, 9-41.

http://www.scielo.org.mx/scielo.php?pid=s0188-77422007000200002\&script=sci_arttext 